\title{
Hippocampal transection for stereo-electroencephalography-proven dominant mesial temporal lobe epilepsy in a child: a detailed case report and critical review
}

\author{
Jun T. Park, MD, ${ }^{1,2}$ Guadalupe Fernandez Baca Vaca, MD, ${ }^{2}$ Rachel Tangen, PhD, ${ }^{3}$ and \\ Jonathan Miller, MD ${ }^{4}$
}

${ }^{1}$ Section of Pediatric Epilepsy, Department of Pediatrics, Rainbow Babies \& Children's Hospital, Case Western University School of Medicine; ${ }^{2}$ Epilepsy Center, Neurological Institute, Department of Neurology, University Hospital, Case Western University

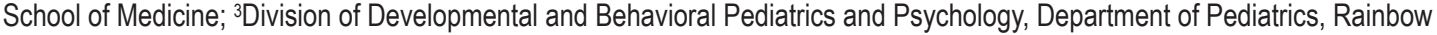
Babies \& Children's Hospital, Case Western University School of Medicine; and "Department of Neurosurgery, Neurological Institute, University Hospital, Case Western University School of Medicine, Cleveland, Ohio

\begin{abstract}
Resection of the hippocampus ipsilateral to the verbal memory-dominant hemisphere frequently results in severe memory deficits. In adults with epilepsy, multiple hippocampal transections (MHTs) have resulted in excellent seizure outcome with preservation of verbal memory. The authors report the first detailed case of a child undergoing MHTs for mesial temporal lobe epilepsy. A 13-year-old right-handed boy had intractable seizures characterized by epigastric discomfort evolving to unresponsiveness and chewing automatisms, lasting 1 minute and occurring 2-3 times weekly, sometimes ending in a generalized tonic-clonic seizure. He had no seizure risk factors and nonfocal examination results. Interictal electroencephalography (EEG) showed frequent left temporal epileptiform discharges (maximum FT9) and intermittent slowing. Video EEG, FDG-PET, and 1.5-T MRI were nonlocalizing. Neuropsychological evaluation suggested left temporal lobe dysfunction. A stereo-EEG investigation using 8 electrodes localized the seizure onset zone to the anterior mesial temporal region, immediately involving the hippocampus. The temporal pole and amygdala were resected en bloc with 3 MHTs. Comparison of neuropsychological tests 4 months before and 6 months after the surgery showed a significant decline only in confrontational naming and no significant change in verbal memory. Six and a half years later, the patient remains seizure free with no antiepileptic drugs. In children with established hemispheric dominance suffering from mesial temporal lobe epilepsy, MHTs may be an option.
\end{abstract}

https://thejns.org/doi/abs/10.3171/2018.5.PEDS1896

KEYWORDS stereotactic electroencephalography; mesial hippocampal transection; dominant temporal lobe epilepsy; pediatric epilepsy surgery; spike synchronization; type B spikes

$\mathrm{T}$ EMPORAL lobe epilepsy is a common cause of focal epilepsy, and resection frequently leads to seizure freedom. ${ }^{6}$ However, resection of the hippocampus ipsilateral to the verbal memory-dominant hemisphere frequently results in severe memory deficits. In adult patients with seizures arising from the mesial temporal lobe, multiple hippocampal transections (MHTs) have been documented to result in excellent seizure outcome with preservation of verbal memory. ${ }^{10}$ We report the first de- tailed case of a child undergoing MHTs for mesial temporal lobe epilepsy.

\section{Case Report}

History and Examination

A 13-year-old right-handed boy began having seizures at 7 years of age. Seizures consisted of epigastric discomfort evolving to unresponsiveness and chewing au-

ABBREVIATIONS AED = antiepileptic drug; ECoG = electrocorticography; EEG = electroencephalography; MSTs = multiple subpial transections; MHTs = multiple hippocampal transections.

SUBMITTED February 26, 2018. ACCEPTED May 15, 2018.

INCLUDE WHEN CITING Published online August 10, 2018; DOI: 10.3171/2018.5.PEDS1896. 
tomatisms lasting 1 minute and occurring 2-3 times per week. Seizures did not typically generalize, but there was a history of 6 generalized tonic-clonic seizures. Four antiepileptic drugs (AEDs) failed, and the patient experienced poor seizure control on levetiracetam. Neurological examination findings were normal, and the patient had normal developmental milestones with no epilepsy risk factors.

\section{Presurgical Evaluation}

Video electroencephalography (EEG) indicated left temporal lobe epilepsy as follows: 1) left temporal sharp waves every 5-10 seconds during sleep, maximum FT9; 2) left temporal spikes every second for 5-8 seconds, maximum FT9; 3) intermittent left frontotemporal slowing lasting 2-8 seconds, $30 \%$ of the record; and 4) epileptic seizures off AEDs: dialepsis followed by right head version (lateralizing sign to the left) followed by generalized tonic-clonic seizure, with EEG onset unclear. FDG-PET provided no lateralization. MRI at $1.5 \mathrm{~T}$ showed volume loss of the right amygdala and hippocampal head without signal abnormality (Fig. 1). This MRI abnormality did not translate to right-sided epileptogenicity. Neuropsychological evaluation showed average to above average cognitive abilities with mild weakness in memory and verbal IQ suggestive of left temporal lobe dysfunction.

\section{Invasive Investigation}

After a discussion at a comprehensive epilepsy surgery conference, a stereo-EEG investigation of the left hemisphere was performed using 8 electrodes to sample the following regions potentially involved in seizure onset (Fig. 2): frontal basal; anterior temporal; amygdala; and hippocampal head, body, and tail. Intended electrode tar- gets were confirmed postoperatively using Brainlab software.

Spikes were recorded exclusively in the hippocampus, amygdala, and temporal pole. This correlated with simultaneous scalp EEG that showed a typical sharp wave over the anterior temporal (F7) region. The patient had a habitual seizure characterized by tachycardia (60-90 $\mathrm{bpm})$, which progressed to hand and mouth automatisms and unresponsiveness. Clinical onset appeared several seconds after EEG seizure pattern reflected in the depth electrodes. The stereo-EEG localized the seizure onset to the anterior mesial temporal region that quickly spread to the hippocampal head, body, and tail. Ictal direct current shift was noted in the same regions: hippocampal head, body, and tail (Fig. 3). With parental consent, the decision was made to perform MHTs to preserve verbal memory. The concept and utility of MHTs have been previously described by Shimizu et al. ${ }^{10}$

\section{Operation}

With the assistance of frameless stereotaxy, the left temporal pole, $3 \mathrm{~cm}$ from the tip, and amygdala were resected en bloc. The hippocampus was accessed through the middle temporal gyrus. The temporal horn was entered, and electrodes were inserted 1-2 $\mathrm{mm}$ deep through the lateral ventricle. Six recording needles (H1-6) were implanted into the head and body of the left hippocampus at 5-mm intervals as follows: electrode numbers 1-2 in the head, 3 at the head/body junction, and 4-6 in the body. The reference electrode for the ipsilateral temporal recordings (approximately 9 minutes) was selected to count the spike population in the cornu ammonis at baseline. In the hippocampus, recording was performed for
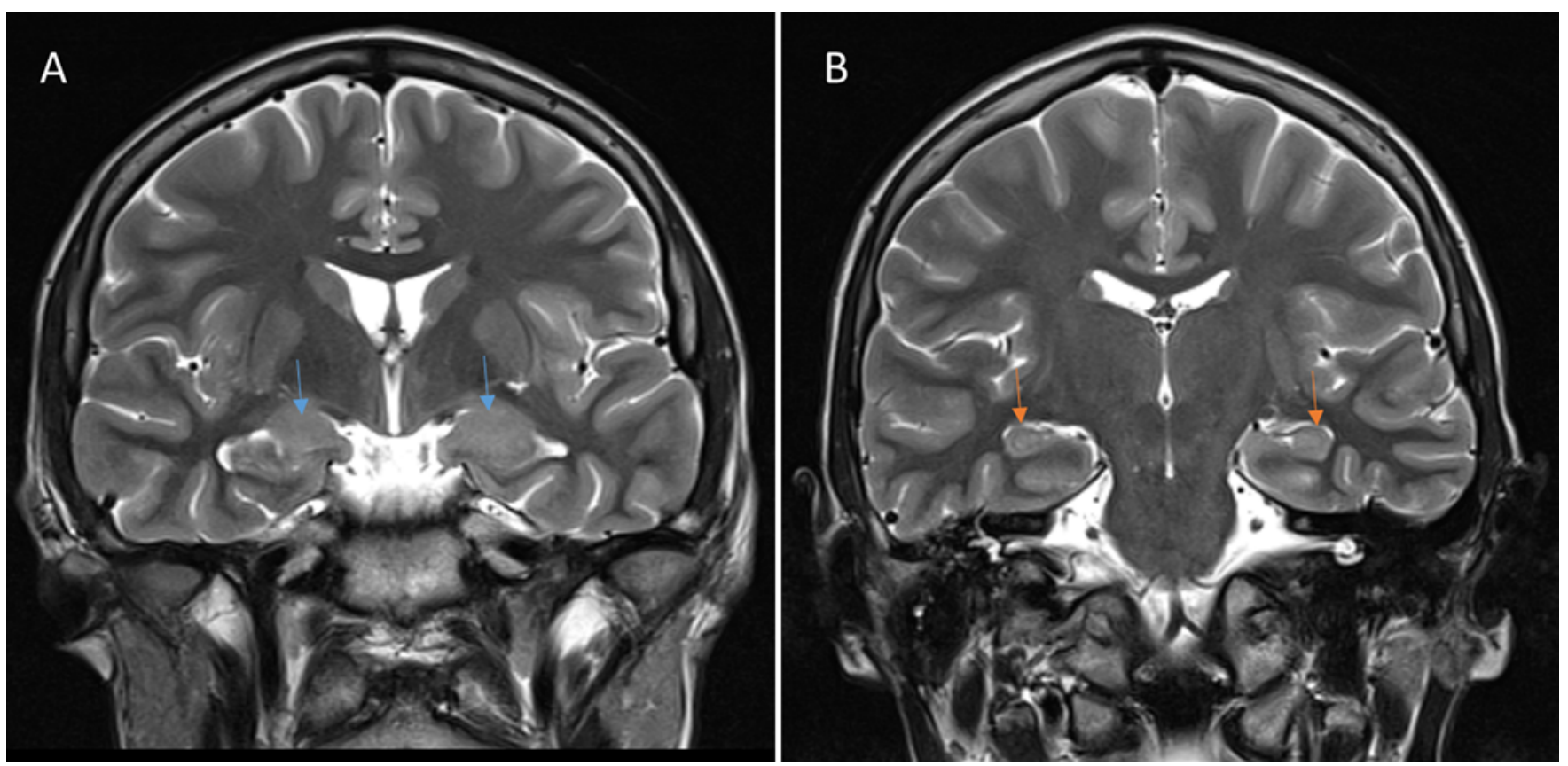

FIG. 1. Preoperative coronal Gd-enhanced T2-weighted MR images of the brain. A: The blue arrows point to the asymmetrical amygdala, with the left being bigger than the right. B: The orange arrows point to the symmetric body of the hippocampus. Figure is available in color online only. 

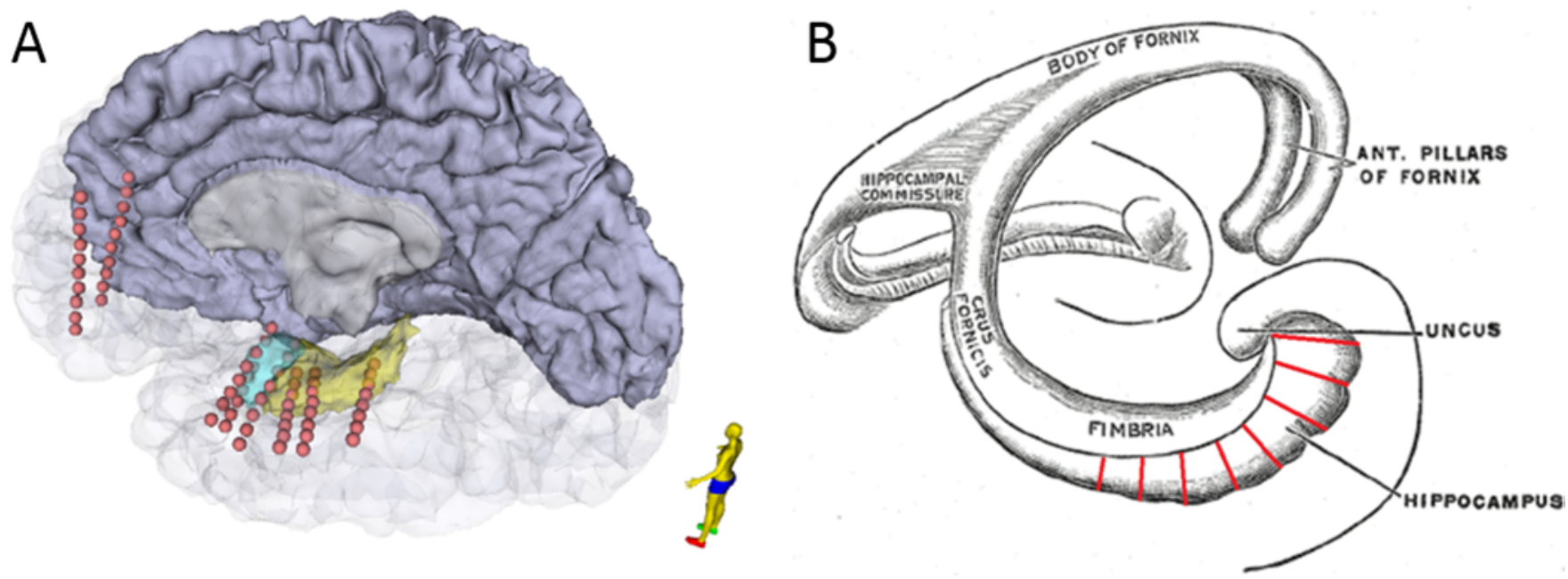

FIG. 2. A: MRI cortical reconstruction and volumetric segmentations were performed using Freesurfer image analysis suite (http:// surfer.nmr.mgh.harvard.edu/). To anatomically overlay electrodes from postoperative CT scans to MR images, we used FLIRT linear registration available in FSL v5.0.9 (https://fsl.fmrib.ox.ac.uk/fsl/). The resulting 3D reconstructed images from Freesurfer and FSL registrations were viewed using a 3D slicer (http://www.slicer.org) with thresholds to remove regions of no interest. Electrode locations in the left hemisphere: frontal basal (lateral frontal and medial frontal, 2 electrodes), anterior temporal (1), amygdala (2), hippocampal head (1), hippocampal body (1), and hippocampal tail (1). (Image: courtesy of Johnson Hampson, an engineer at Cleveland University Hospitals). B: Schematic of the hippocampus showing transections (red). Figure 747 from Gray H. Anatomy of the Human Body, Lewis WH (ed). Philadelphia: Lea and Febiger, 1918. Figure is available in color online only.

15 minutes to identify the location where the frequency and amplitude of epileptiform discharges were greatest. Subsequently, 10 minutes of counting spikes and attention to amplitudes preceded each cut. The surface of the hippocampus was opened sharply along the cornu ammonis, and a custom-made 2-mm steel wire loop was inserted through the hippocampus as far as the mesial pia, taking care to avoid the alveus. The first cut was made between electrodes $\mathrm{H} 3$ and $\mathrm{H} 4$, followed by a cut between $\mathrm{H} 4$ and $\mathrm{H} 5$, followed by a final cut between $\mathrm{H} 2$ and $\mathrm{H} 3$. After each cut, there was an observable reduction in spike frequency, followed by synchronization of spikes and polyspikes, and finally reduced spike amplitude (Fig. 2). The epileptiform discharges were not eliminated after the last transection, although they were visibly reduced in frequency, amplitude, and synchronization. The extent of the transection and whether the transection involved the subiculum were unknown, although the extrahippocampal structures were preserved, including the fimbria since it represents the main outflow pathway of the hippocampus. Further details of the operation were published by another group at our institution. ${ }^{8}$

\section{Neuropsychological Evaluations}

A comprehensive battery of standardized neuropsychological tests was administered 4 months before and 6 months after the surgery. The pre- and postsurgical test scores were compared using the reliable change index with an adjustment for practice effects ${ }^{4}$ and applied to a pediatric epilepsy case as described by Busch et al. ${ }^{3}$ Most notably, there was no significant change in verbal memory compared with preoperative testing. Children's Memory Scale visual immediate and delayed memory did not change. Intellectual skills remained in the average range without significant change in verbal or nonverbal scores. Language scores were variable as phonemic verbal fluency significantly improved, but there was a significant decline in confrontational naming. No other cognitive abnormalities were reported after the operation (Table 1). Since the surgery, the patient has remained seizure free with no antiepileptic medication and is attending college.

\section{Discussion}

When Shimizu et al. first reported on 17 patients who underwent MHTs,${ }^{10}$ they applied the rationale of multiple subpial transections (MSTs) to the hippocampus. That group monitored these patients for over a year and reported excellent seizure and memory outcome. Since that report, supporting data have been published that shed light on the anatomical and functional organization of the hippocampus. The hippocampus contains 2 types of pathways: 1 ) trisynapatic pathways that are arranged in parallel loops, perpendicular to the longitudinal axis of the hippocampus; and 2) longitudinal pathways that run across the long axis of the hippocampus. ${ }^{2}$ The loops of trisynaptic pathways, originating and ending in the entorhinal cortex, are important for memory processing, ${ }^{1}$ while the longitudinal pathways are important for synchronization of epilepsy discharges..$^{12}$ The information on the organization of the hippocampus and the favorable outcome reported by Shimizu et al. helped to establish a rationale for MHTs in the selected patients with dominant mesial temporal lobe epilepsy. ${ }^{10}$ More recently, numerous groups of investigators have reported excellent seizure and memory outcomes in patients who underwent MHTs. ${ }^{8,9,11,12}$

Temporal lobe epilepsy is not rare in children. In the very young child who has yet to establish hemispheric 


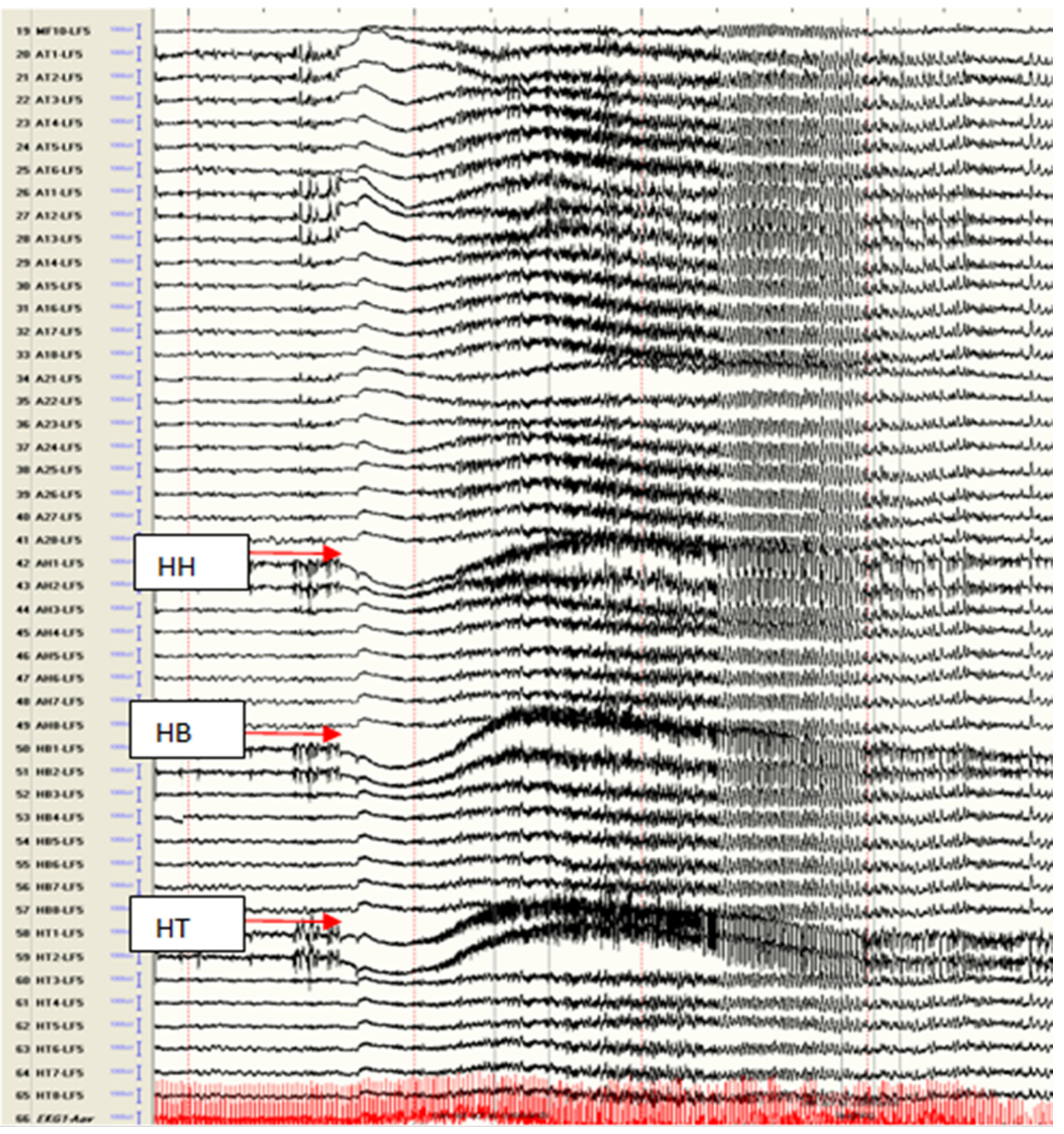

FIG. 3. EEG recordings during a seizure. The red arrows indicate ictal direct current shift over the hippocampal head (HH), body

$(\mathrm{HB})$, and tail $(\mathrm{HT})$. Figure is available in color online only.

dominance, temporal lobe resection can be safely done on either side. However, once hemispheric dominance is established, hippocampal resection on the dominant side can result in major memory deficits.

This is the first detailed report of a child who underwent MHTs for stereo-EEG-proven mesial temporal lobe epilepsy with a follow-up duration of 6.5 years. Two sepa- rate case series from Japan documented results of multiple tests before and after surgery as part of a detailed follow-up evaluation, but most patients in both of these reports were adults. Shimizu et al. ${ }^{10}$ studied 21 patients, 2 of whom were children (ages 2 and 9 years), who underwent left hippocampal transection plus MSTs with or without resection of the amygdala and temporal tip. Neu- 
TABLE 1. Preoperative and postoperative standard scores, practice effects, and adjusted reliable change index scores for neuropsychological measures

\begin{tabular}{|c|c|c|c|c|c|}
\hline Neuropsychological Measure & $\begin{array}{c}\text { Preop Standard } \\
\text { Score* }\end{array}$ & $\begin{array}{l}\text { Postop Standard } \\
\text { Score }^{*}\end{array}$ & $\begin{array}{l}\text { Practice } \\
\text { Effect† }\end{array}$ & Adjusted RCI 90\% Cl $\ddagger$ & $\begin{array}{c}\text { Adjusted } \\
\text { RCl }\end{array}$ \\
\hline WISC-IV full scale IQ & 108 & 105 & 1 & -10 to 12 & -0.62 \\
\hline WISC-IV verbal comprehension index & 98 & 96 & -2 & -16 to 12 & 0.0 \\
\hline WISC-IV perceptual reasoning index & 110 & 104 & 2 & -14 to 17 & -0.82 \\
\hline WISC-IV working memory index & 110 & 102 & 3 & -18 to 23 & -0.87 \\
\hline WISC-IV processing speed index & 109 & 115 & 0 & -16 to 17 & 0.60 \\
\hline COWA (FAS) phonemic fluency & 104 & 120 & 1 & -7 to 9 & 3.00 \\
\hline Boston Naming Test & 85 & 73 & 1 & -4 to 6 & -2.83 \\
\hline CMS verbal immediate memory§ & 97 & 103 & 6 & -11 to 22 & 0.0 \\
\hline CMS verbal delayed memory§ & 100 & 100 & 8 & -14 to 30 & -0.62 \\
\hline CMS visual immediate memory & 97 & 122 & 10 & -11 to 31 & 1.20 \\
\hline CMS visual delayed memory & 106 & 118 & 11 & -10 to 33 & -0.08 \\
\hline CVLT-C total learning ${ }^{* *}$ & 97 & 94 & 6 & -4 to 16 & -1.54 \\
\hline CVLT-C Short Delay Recall** & 100 & 100 & 2 & -1 to 5 & -1.21 \\
\hline
\end{tabular}

CMS = Children's Memory Scale; COWA = Controlled Oral Word Association Test; CVLT-C = California Verbal Learning Test-Children's Version; RCI = reliable change index; WISC-IV = Wechsler Intelligence Scale for Children-Fourth Edition.

Boldface type indicates a statistically significant change between time points of scores greater than 1.64 or less than -1.64 at a 0.10 significance level $(90 \% \mathrm{Cl})$.

${ }^{*}$ Scores on these measures are reported as standard scores with a mean of 100 and SD of 15.

† Practice effect scores from pediatric epilepsy sample (11-16 years old) as reported in Busch et al. 2015, when available, or other normative sample.

$\ddagger$ The adjusted reliable change index equals the [postoperative score - preoperative score] - practice effect/standard error of the difference. Measures susceptible to decline after dominant temporal lobe surgery from a larger test battery were analyzed pre-and postoperatively. The $90 \% \mathrm{Cl}$ represents cutoff values at or beyond which an observed change score represents a clinically significant change after adjusting for test reliability and practice effects.

$\S$ The Children's Memory Scale verbal memory index remained in the average range for both immediate and delayed recall.

I The Children's Memory Scale visual immediate memory index improved but not significantly when controlling for practice effects, while the visual delayed memory index remained in the average range.

${ }^{* *}$ Verbal list learning on the California Verbal Learning Test-Children's Version remained in the average range for total list learning and short-term recall.

ropsychological evaluation was not done before or after the surgery in the 9-year-old patient, and seizure freedom for individual patients was not reported during the short follow-up period. No explanation was provided as to why the use of MHTs was preferred over resection in the 2-year-old patient, who had yet to establish hemispheric dominance. In the second case series, Uda et al. ${ }^{11}$ reported on 37 patients, 4 of whom were younger than 16 years, who underwent MHTs plus resection of the inferior part of the amygdala for the purpose of obtaining a surgical field. The children were excluded from the neuropsychological memory evaluation (verbal memory, nonverbal memory, and delayed recall) due to their young age. The patients in that study, who underwent surgery by a single surgeon, were recruited from 2 different institutions in different cities during 2005-2011. It is unknown whether there was an overlap in the recruited patients in the studies. The reported overall seizure freedom rate was $67.6 \%$ in that series. It is unknown whether any of the patients remained on $\operatorname{AED}(\mathrm{s})$ at the time of follow-up.

Compared with the baseline count in our patient, in the previously reported patients approximately $70 \%$ of spikes remained with visibly reduced amplitude and spike synchronization after the third (last) transection, which was after the resection of the amygdala and temporal tip. Our patient has not had a seizure since the surgery and he has been off AEDs for the past 6.5 years. Based on the stereoEEG investigation, we feel confident that the hippocam- pus was the epileptogenic zone. This raises an important question: Is total elimination of spikes in the hippocampus necessary to achieve seizure freedom, regardless of the number of transections performed? Prior studies on outcome after MHTs, although limited, assume that all epileptic potentials in the hippocampus must be abolished to achieve seizure freedom. This notion is an extension from an earlier study supporting the fact that longitudinal connections in hippocampus are involved in seizure synchronization, and that interictal spikes tend to correlate with seizure activity. ${ }^{12}$ Two major series reported that the number of transections of hippocampus needed and whether additional MSTs are necessary are dependent on when the spikes are eliminated on electrocorticography $(\mathrm{ECoG}){ }^{9,10}$ Patients in the study by Shimizu et al. ${ }^{10}$ all had MHTs with or without amygdalectomy and resection of the anterior basal cortex, with subsequent MSTs of the posterior basal area and superficial surface. In comparison, patients in the study by Patil and Andrews ${ }^{9}$ all had MHTs with or without amygdalectomy, temporal tip resection, and subsequent MSTs of the lateral and basal surfaces of the temporal lobe, including the parahippocampus and entorhinal cortex. Seizure freedoms of $82 \%$ and $94.7 \%$ (Engel class I) were reported in the 2 studies. As shown in these studies, MHTs are rarely done alone without subsequently "chasing" spikes so that ultimately additional MSTs of basal and lateral temporal neocortices are done as well as resection of additional regions if needed. Thus, it is difficult to 


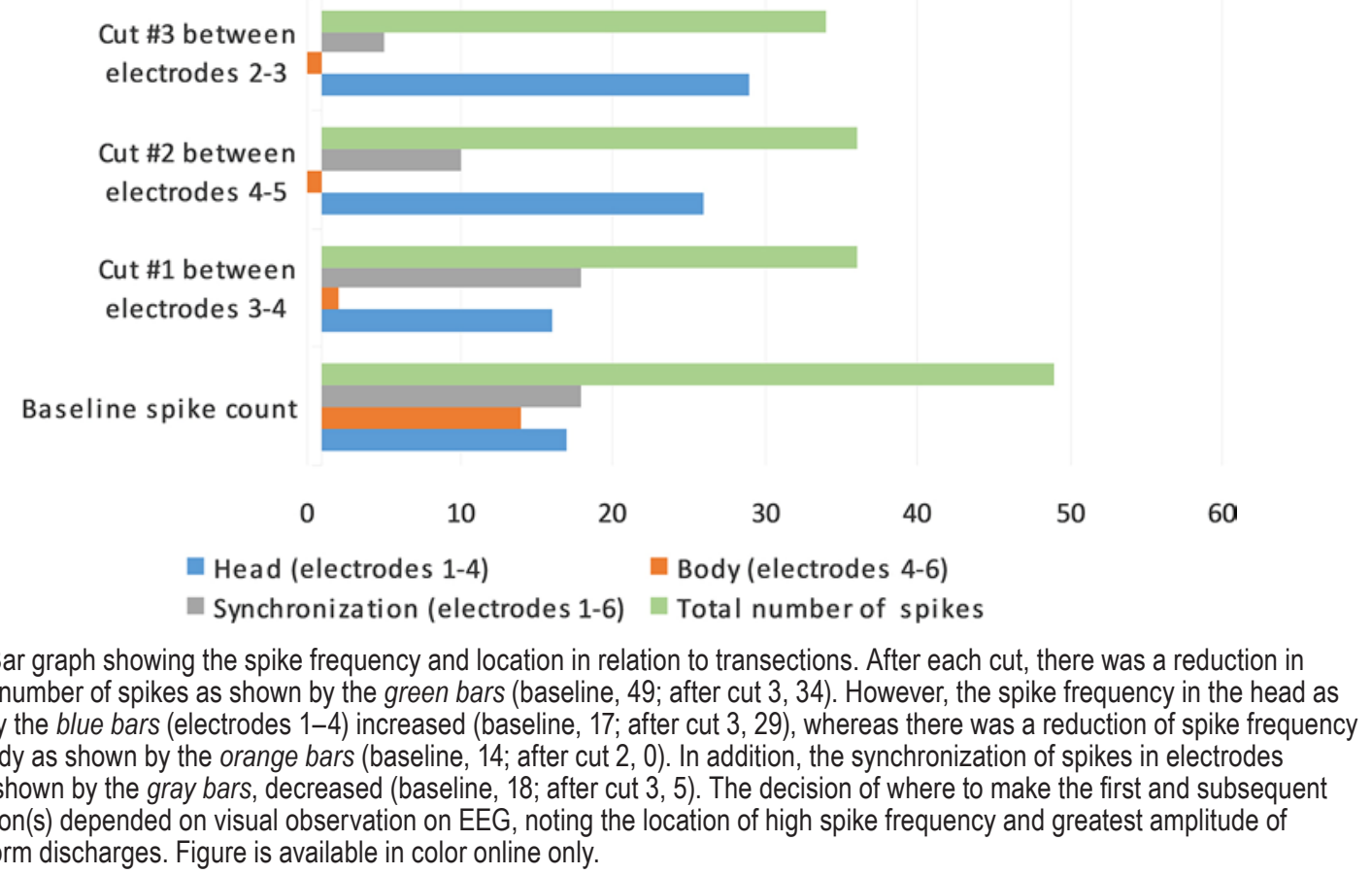

prove when the patient becomes seizure free whether the epileptogenic zone was in fact the hippocampus.

If the epileptogenic zone is the hippocampus and MHTs are effective, this procedure alone should result in seizure freedom, as suggested by this case study. Two studies of MHTs in adults extend this conclusion. In the study by Uda et al., ${ }^{11} 22$ adult patientswith a mean follow-up duration of 40 months reported elimination of all active epileptic discharges over the hippocampus after MHTs and amygdala resection. They reported an Engel class I seizure freedom rate of $67 \%$. It is unknown whether spikes would have remained after the completion of MHTs if needle electrodes had been used instead of subdural ECoG. In another study, Koubeissi et al.$^{8}$ reported on 12 adults, 8 of whom underwent only MHTs (3-6 transections), and the remaining 4 adults had additional resection of the temporal tip and amygdala, one of whom had additional parahippocampus resection. All patients underwent stereo-EEG presurgical evaluation with subsequent needle electrode EcoG evaluation. Four of the 8 patients who only had MHTs were seizure free, while the other 4 achieved Engel class I outcome. Also included in the outcome report was 1 child, who is the patient detailed in this report. The overall seizure Engel class I outcome was $83 \%$. The authors reported a reduction of spike frequency by over $60 \%$ after the third transection. However, no details were available as to whether all spikes were eliminated by the end of the last transection. Comparison of MHTs done with and without resection of the amygdala and temporal tip in patients with stereo-EEG-confirmed mesial temporal lobe epilepsy has revealed no difference in either seizure or memory outcome. ${ }^{7}$ It is important to note that studies that have reported elimination of spikes in the hippocampus with MHTs and additional MSTs of temporal neocortices used subdural ECoG. Because the hippocampus is a closed circuit, ${ }^{5}$ recording from the surface of the hippocampus may not record spikes that might be seen using needle electrodes.

In a pilot study by Umeoka et al. ${ }^{12}$ examining the role of longitudinal hippocampal circuits in the generation of interictal and ictal activity and the effects of MHTs in temporal lobe epilepsy, 1-2 transections between the head and body of hippocampus were found to be sufficient to abolish a specific type of synchronized spikes (type B spikes, maximum amplitude over body of hippocampus) to render a patient seizure free. However, the other type of hippocampal spikes (type $\mathrm{H}$, maximum amplitude over the hippocampal head) showed no changes in amplitude and distribution, and essentially unchanged in frequency during and after the transections. Thus, the study concluded that well-synchronized hippocampal spikes, i.e., type B spikes, with an anteroposterior propagation pattern are important for seizure generation in mesial temporal lobe epilepsy. Therefore, elimination of all hippocampal spikes was not supportive for seizure freedom, as seen in our patient. ECoG data analysis in our patient showed that spike frequency in the head of the hippocampus increased, while no spikes were seen in the body after the second cut. Synchronized spikes involving all 6 electrodes also decreased in frequency from 18 to 5 after the last cut (Fig. 4, Table 2 ). We therefore propose that the number of transections of the hippocampus needed for a favorable outcome primarily depends on when the type B spikes are eliminated. This is also supported by unpublished data at out center where an adult patient with left hippocampal epilepsy became seizure free for at least 2 years after only 1 cut in the posterior hippocampal region based on the location of spikes with highest amplitude.

In children with established hemispheric dominance and mesial temporal lobe epilepsy, MHTs may be an option with excellent memory and seizure outcome. No stan- 
TABLE 2. Spike frequency and location in relation to transections

\begin{tabular}{lccccc}
\hline & \multicolumn{3}{c}{ Hippocampus } & & \\
\cline { 2 - 3 } & Head (electrodes 1-4) & Body (electrodes 4-6) & Synchronization (electrodes 1-6) & Total No. of Spikes \\
\hline Baseline spike count & 17 & 14 & 18 & 49 \\
\hline Cut 1 btwn electrodes 3 \& 4 & 16 & 2 & 18 & 36 \\
\hline Cut 2 btwn electrodes 4 \& 5 & 26 & 0 & 10 & 36 \\
\hline Cut 3 btwn electrodes 2 \& 3 & 29 & 0 & 5 & 34 \\
\hline
\end{tabular}

dardization of MHTs exists to date, although some variations in techniques may be possible. The goal of eliminating all hippocampal spikes may be difficult to achieve, even when using needle electrodes during ECoG. Rather, the aim of hippocampal MHTs should be to eliminate hippocampal spikes that are synchronized along the anteroposterior axis, as originally described by Umeoka et al. ${ }^{12}$ A multicenter clinical study using standardized techniques and long-term follow-up studies that include a comparison of detailed neuropsychological evaluations before and after the standardized MHTs will enhance understanding of outcome in these children.

\section{References}

1. Acsády L, Káli S: Models, structure, function: the transformation of cortical signals in the dentate gyrus. Prog Brain Res 163:577-599, 2007

2. Amaral DG, Witter MP: The three-dimensional organization of the hippocampal formation: a review of anatomical data. Neuroscience 31:571-591, 1989

3. Busch RM, Lineweaver TT, Ferguson L, Haut JS: Reliable change indices and standardized regression-based change score norms for evaluating neuropsychological change in children with epilepsy. Epilepsy Behav 47:45-54, 2015

4. Chelune GJ, Naugle RI, Lüders H, Sedlak J, Awad IA: Individual change after epilepsy surgery: practice effects and base-rate information. Neuropsychology 7:41-52, 1993

5. Deadwyler SA, West JR, Cotman CW, Lynch G: Physiological studies of the reciprocal connections between the hippocampus and entorhinal cortex. Exp Neurol 49:35-57, 1975

6. Engel J Jr, McDermott MP, Wiebe S, Langfitt JT, Stern JM, Dewar S, et al: Early surgical therapy for drug-resistant temporal lobe epilepsy: a randomized trial. JAMA 307:922-930, 2012

7. Girgis F, Greil ME, Fastenau PS, Sweet J, Lüders H, Miller JP: Resection of temporal neocortex during multiple hippocampal transections for mesial temporal lobe epilepsy does not affect seizure or memory outcome. Oper Neurosurg (Hagerstown) 13:711-717, 2017

8. Koubeissi MZ, Kahriman E, Fastenau P, Bailey C, Syed T, Amina S, et al: Multiple hippocampal transections for intractable hippocampal epilepsy: seizure outcome. Epilepsy Behav 58:86-90, 2016
9. Patil AA, Andrews R: Long term follow-up after multiple hippocampal transection (MHT). Seizure 22:731-734, 2013

10. Shimizu H, Kawai K, Sunaga S, Sugano H, Yamada T: Hippocampal transection for treatment of left temporal lobe epilepsy with preservation of verbal memory. J Clin Neurosci 13:322-328, 2006

11. Uda T, Morino M, Ito H, Minami N, Hosono A, Nagai T, et al: Transsylvian hippocampal transection for mesial temporal lobe epilepsy: surgical indications, procedure, and postoperative seizure and memory outcomes. J Neurosurg 119:10981094,2013

12. Umeoka SC, Lüders HO, Turnbull JP, Koubeissi MZ, Maciunas RJ: Requirement of longitudinal synchrony of epileptiform discharges in the hippocampus for seizure generation: a pilot study. J Neurosurg 116:513-524, 2012

\section{Disclosures}

The authors report no conflict of interest concerning the materials or methods used in this study or the findings specified in this paper.

\section{Author Contributions}

Conception and design: Park. Acquisition of data: Park, Miller. Analysis and interpretation of data: Park, Fernandez Baca Vaca, Tangen. Drafting the article: Park, Fernandez Baca Vaca, Tangen. Critically revising the article: Park, Fernandez Baca Vaca, Miller. Reviewed submitted version of manuscript: Park, Miller. Approved the final version of the manuscript on behalf of all authors: Park. Statistical analysis: Tangen.

\section{Supplemental Information}

\section{Previous Presentations}

Portions of this paper were presented in abstract form at the 71st American Epilepsy Society Annual Meeting, Washington, DC, December 1-5, 2017.

\section{Correspondence}

Jun T. Park: Rainbow Babies \& Children's Hospital, Case Western University School of Medicine, Cleveland, OH. jun.park@ uhhospitals.org. 